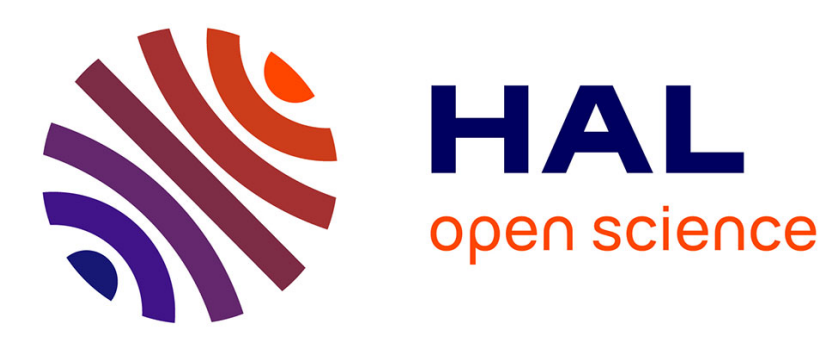

\title{
Extraction of tori from minimal point sets
}

\author{
Laurent Busé, André Galligo
}

\section{To cite this version:}

Laurent Busé, André Galligo. Extraction of tori from minimal point sets. Computer Aided Geometric Design, 2017, 58, pp.1 - 7. 10.1016/j.cagd.2017.10.004 . hal-01553065v2

\section{HAL Id: hal-01553065 \\ https://hal.inria.fr/hal-01553065v2}

Submitted on 8 Dec 2017

HAL is a multi-disciplinary open access archive for the deposit and dissemination of scientific research documents, whether they are published or not. The documents may come from teaching and research institutions in France or abroad, or from public or private research centers.
L'archive ouverte pluridisciplinaire HAL, est destinée au dépôt et à la diffusion de documents scientifiques de niveau recherche, publiés ou non, émanant des établissements d'enseignement et de recherche français ou étrangers, des laboratoires publics ou privés. 


\title{
Extraction of tori from minimal point sets
}

\author{
Laurent Busé ${ }^{1}$ and André Galligo ${ }^{2}$ \\ ${ }^{1}$ Université Côte d'Azur, Inria, France. \\ ${ }^{2}$ Université Côte d'Azur, Laboratoire J.-A. Dieudonné and Inria, France.
}

September 14, 2017

\begin{abstract}
A new algebraic method for extracting tori from a minimal point set, made of two oriented points and a simple point, is proposed. We prove a degree bound on the number of such tori; this bound is reached on examples, even when we restrict to smooth tori. Our method is based on pre-computed closed formulae well suited for numerical computations with approximate input data.
\end{abstract}

Keywords: mixed set of 3D points, tori, interpolation method.

\section{Introduction}

The extraction of geometric primitives from 3D point clouds is an important problem in reverse engineering. These 3D point clouds are typically obtained by means of accurate 3D scanners and there exist several methods for performing the 3D geometric primitives extraction. An important category among them are the statistical and iterative methods based on the RANSAC (RANdom SAmple Consensus) paradigm [3, 7, 8]. Key ingredients in this approach are geometric routines that are capable to produce an instance of a given type of shape from a small number of points. For instance, computing the equation of a plane passing through three given points, or passing through a point-with-normal (a point with a normal vector), are basic routines that are intensively used in RANSAC-based methods. In practice, the most used types of shapes are planes, spheres, cylinders, cones and tori. While devising such routines is relatively straightforward for planes and spheres, the cases of cylinders, cones and tori are much more difficult. In a previous work [1] we proposed a detailed analysis and efficient algorithms for cylinders and cones. In this note we treat the case of tori. More precisely, we provide a new method for extracting tori from the smallest possible number of conditions, that is to say from two point-with-normal and a single point, which account for seven parameters, i.e. the same number as the degrees of freedom of the considered interpolation problem. We emphasize that it is very important to compute shapes from the smallest possible number of conditions in order to guarantee efficiency and accuracy in these interpolation processes. Most of the methods that are currently used in practical applications for cylinders, cones and tori are based on the solving of overdetermined linear systems so that the computed shapes are not interpolating the point-with-normal data but are only approximating them.

In the sequel, an oriented point is a couple of a point and a nonzero vector. A surface is said to interpolate an oriented point if the point belongs to the surface and its associated vector is colinear to the normal of the surface at this point. Notice that we are not assuming that the orientation of the normal of the point is the same as the orientation of the surface. Moreover, it is important to deal with inhomogeneous data, that is to say some points are oriented but not all, in order to take into account the estimated accuracy of oriented point clouds that are obtained by means of normal estimation algorithms. A set of data made of points and oriented points is called a mixed set of points. 
Previous methods for the extraction of tori from a small number of points in a RANSAC-like approach have been treated from an overdetermined number of conditions, i.e. mixed set of points that are bigger than necessary, which has the consequence that computed tori are not exactly interpolating the data. For instance, in [6] tori are approximated from four oriented points, i.e. twelve conditions, and in $[4,5]$ tori are approximated from three oriented points, i.e. nine conditions. In this note, we propose a new method that interpolate tori from two oriented points and a single point, i.e. from seven conditions, which is precisely the number of parameters needed to instantiate a torus. At the heart of this contribution is an original and subtle modelisation of this interpolation problem, in opposition to the brute force approach that leads to huge and time consuming treatments of polynomial systems of equations of degree 4 in 7 unknows. Based on this modelisation, our approach relies on adapted algebraic techniques and allows to develop an efficient interpolation algorithm in the context of numerical computations in double precision with approximate data. As a byproduct, we will also get the following theorem of enumerative geometry which seems to be unknown.

Theorem. There exist at most eight non-degenerated tori (or an infinity) that interpolate three distinct points, two of them being oriented.

Our strategy of proof relies on geometric constructions related to 3D interpolation of circles which are described in Section 2. Our new torus interpolation method is developed in Section 3. In Section 4, we will report on our numerical experiments, and also show an example for which this upper bound (eight) is reached, even when we restrict to smooth tori.

\section{$23 \mathrm{D}$ circles passing through two oriented points}

The spine of a torus is a 3D circle. Such a circle depends on six parameters, namely three parameters for the coordinates of its center, two parameters for its supporting plane and one last parameter corresponding to its radius. Thus 3D circles and pairs of oriented points share the same number of degrees of freedom, namely six, and hence one may ask how many circles pass through two oriented points.

We recall that a vector $V$ is said normal to a $3 \mathrm{D}$ curve $\mathcal{C}$ at a smooth point $M$ when $V$ is orthogonal to the tangent vector to $\mathcal{C}$ at $M$.

Proposition 1. We suppose that two distinct points $A_{1}$ and $A_{2}$ and two nonzero vectors $N_{1}$ and $N_{2}$ are given. For $i=1,2$, we denote by $\Omega_{i}$ the intersection point, possibly at infinity, between the bisecting plane of the segment $\left[A_{1} A_{2}\right]$ and the line passing through $A_{i}$ and parallel to $N_{i}$ (see Figure 1). Consider the following fitting problem $(P)$ : determine all the $3 D$ circles that interpolate $A_{1}$ and $A_{2}$ and which are normal to $N_{1}$ at $A_{1}$ and normal to $N_{2}$ at $A_{2}$.

(a) If $\Omega_{1} \neq \Omega_{2}$ and $\Omega_{1}, \Omega_{2}$ are not both at infinity, then there is one and only one circle that satisfies $(P)$.

(b) If $\Omega_{1} \neq \Omega_{2}$ and $\Omega_{1}, \Omega_{2}$ are both at infinity, then there is no circle that satisfies $(P)$.

(c) If $\Omega_{1}=\Omega_{2}$ then there are infinitely many circles that satisfy $(P)$.

Proof. First, we observe that a 3D circle $\mathcal{C}$ defines a sheaf of spheres whose centers belong to the line $L$ passing by the center of $\mathcal{C}$ and orthogonal to its supporting plane. Suppose that a point $A$ on $\mathcal{C}$ and a nonzero vector $N$ are given. Denote by $D$ the line through $A$ and parallel to $N$. Then, $N$ is orthogonal to $\mathcal{C}$ at $A$ if and only if $N$ is orthogonal at $A$ to one and only one of the spheres of the sheaf associated to $\mathcal{C}$, namely the one whose center is the intersection point between the lines $D$ and $L$. We notice that if $D$ and $L$ are parallel lines, i.e. if $N$ is normal to the supporting plane of $\mathcal{C}$, then the corresponding "limit sphere" of the sheaf of spheres has to be seen as the supporting plane of $\mathcal{C}$, since its center is at infinity and its radius is infinite.

Now, returning to our fitting problem, let $\mathcal{C}$ be a circle that interpolates the two distinct points $A_{1}$ and $A_{2}$. By the previous observation, we have that the vector $N_{1}$ is normal to $\mathcal{C}$ at $A_{1}$ if and only if $N_{1}$ is normal at $A_{1}$ to the sphere $S_{1}$ whose center is $\Omega_{1}$ and that goes through $A_{1}$. In other words, the vector $N_{1}$ is normal to $\mathcal{C}$ at $A_{1}$ if and only if $\mathcal{C}$ is contained in the sphere $S_{1}$. Similarly, the vector $N_{2}$ is normal to $\mathcal{C}$ at $A_{2}$ if and 
only if $\mathcal{C}$ is contained in the sphere $S_{2}$ whose center is $\Omega_{2}$ and that goes through $A_{2}$. We recall that if $\Omega_{1}$, respectively $\Omega_{2}$, is at infinity then $S_{1}$, respectively $S_{2}$, is the normal plane to $N_{1}$ through $A_{1}$, respectively the normal plane to $N_{2}$ through $A_{2}$.

To conclude the proof we see that if $\Omega_{1} \neq \Omega_{2}$ and $\Omega_{1}, \Omega_{2}$ are not both at infinity then the intersection of $S_{1}$ and $S_{2}$ defines a unique circle because this is the intersection of two spheres, or a sphere and a plane, which contains the two distinct points $A_{1}$ and $A_{2}$. If $\Omega_{1} \neq \Omega_{2}$ and $\Omega_{1}, \Omega_{2}$ are both at infinity then $S_{1}$ and $S_{2}$ are two distinct planes whose intersection is the line through $A_{1}$ and $A_{2}$, so there is no solution to (P) in this case. Finally, if $\Omega_{1}=\Omega_{2}$ then $S_{1}$ and $S_{2}$ are both the same sphere, or the same plane, and hence any circle on this sphere, or on this plane, that goes through $A_{1}$ and $A_{2}$ will give a solution to $(\mathrm{P})$.

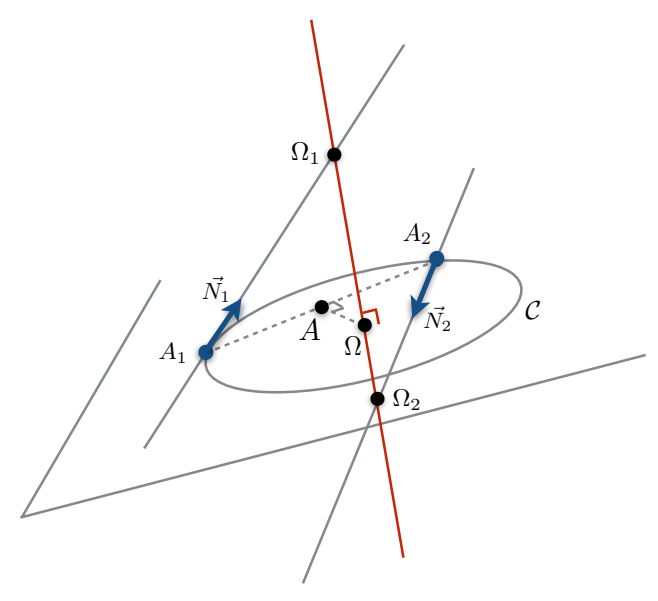

Figure 1: Fitting a 3D circle passing through two distinct points with normal directions.

The above geometric constructions can be quantified to provide explicit formulae that we will use in the next section. Without loss of generality, one may assume that $N_{1}$ and $N_{2}$ are unitary vectors, which we will do in this section.

Case $\left(a_{1}\right)$. We begin with a sub-case of the first item of Proposition 1, that we denote $\left(a_{1}\right): \Omega_{1} \neq \Omega_{2}$ and neither $\Omega_{1}$ nor $\Omega_{2}$ is at infinity. Let $\Omega_{1}:=A_{1}+\lambda_{1} N_{1}$ and $\Omega_{2}:=A_{2}+\lambda_{2} N_{2}$. We have $\left\|\Omega_{1} A_{1}\right\|^{2}=\left\|\Omega_{1} A_{2}\right\|^{2}$ and $\left\|\Omega_{2} A_{1}\right\|^{2}=\left\|\Omega_{2} A_{2}\right\|^{2}$. Let $A$ denotes the middle of $A_{1}$ and $A_{2}$ (see Figure 1 ). Since the plane $\left(A, \Omega_{1}, \Omega_{2}\right)$ is the plane of symmetry of the segment $\left[A_{1}, A_{2}\right]$, we have $\overrightarrow{\Omega_{1} A} \cdot \overrightarrow{A_{1} A_{2}}=0$ and $\overrightarrow{\Omega_{1} \Omega} \cdot \overrightarrow{A_{1} A_{2}}=0$. We deduce that $\left(\overrightarrow{\Omega_{1} A_{1}}+\frac{1}{2} \overrightarrow{A_{1} A_{2}}\right) \cdot \overrightarrow{A_{1} A_{2}}=0$ and we get

$$
\lambda_{1}=\frac{\left\|A_{1} A_{2}\right\|^{2}}{2 \overrightarrow{N_{1}} \cdot \overrightarrow{A_{1} A_{2}}}, \lambda_{2}=\frac{\left\|A_{1} A_{2}\right\|^{2}}{2 \overrightarrow{N_{2}} \cdot \overrightarrow{A_{2} A_{1}}} .
$$

Now, the center $\Omega$ of the circle $\mathcal{C}$ satisfies $\left\|\Omega \Omega_{1}\right\|^{2}=\left\|\Omega_{1} A_{1}\right\|^{2}-\left\|A_{1} \Omega\right\|^{2}=\lambda_{1}^{2}-R^{2}$, and similarly for $\Omega_{2}$, where $R$ stands for the radius of $\mathcal{C}$. Substituting in the expression of the squared norm of $\overrightarrow{\Omega_{2}}=\overrightarrow{\Omega_{\Omega_{1}}}+\overrightarrow{\Omega_{1} \Omega_{2}}$, we obtain $\lambda_{2}^{2}-R^{2}=\lambda_{1}^{2}-R^{2}+\left\|\Omega_{1} \Omega_{2}\right\|^{2}+2 \overrightarrow{\Omega \Omega_{1}} \cdot \overrightarrow{\Omega_{1} \Omega_{2}}$ and we deduce

$$
\Omega=\Omega_{1}+\frac{1}{2}\left(\frac{\left(\lambda_{1}^{2}-\lambda_{2}^{2}\right)}{\left\|\Omega_{1} \Omega_{2}\right\|^{2}}+1\right) \overrightarrow{\Omega_{1} \Omega_{2}}, R^{2}=\lambda_{1}^{2}-\frac{1}{4} \frac{\left(\lambda_{1}^{2}-\lambda_{2}^{2}+\left\|\Omega_{1} \Omega_{2}\right\|^{2}\right)^{2}}{\left\|\Omega_{1} \Omega_{2}\right\|^{2}} .
$$

Symmetrizing these formulae we get

$$
\Omega=\frac{\Omega_{1}+\Omega_{2}}{2}+\frac{\left(\lambda_{1}^{2}-\lambda_{2}^{2}\right)}{2} \frac{\overrightarrow{\Omega_{1} \Omega_{2}}}{\left\|\Omega_{1} \Omega_{2}\right\|^{2}}, 4 R^{2}=2\left(\lambda_{1}^{2}+\lambda_{2}^{2}\right)-\left\|\Omega_{1} \Omega_{2}\right\|^{2}-\frac{\left(\lambda_{1}^{2}-\lambda_{2}^{2}\right)^{2}}{\left\|\Omega_{1} \Omega_{2}\right\|^{2}} .
$$


Expressing the previous formulae with coordinates reveals unexpected algebraic properties which will be useful in the next section. Choosing an adapted frame, we can let $A_{1}$ be the origin, $N_{1}=(0,0,1)$, $N_{2}=(l, m, n)$, and $A_{2}=\left(0, y_{2}, z_{2}\right)$. It follows that

$$
\left\|A_{1} A_{2}\right\|^{2}=y_{2}^{2}+z_{2}^{2}, \overrightarrow{N_{1}} \cdot \overrightarrow{A_{1} A_{2}}=z_{2}, \overrightarrow{N_{2}} \cdot \overrightarrow{A_{2} A_{1}}=m y_{2}+n z_{2} .
$$

Then, simple formal computations which could be done by hand but are easier with a computer algebra system yields the following properties.

Lemma 2. Using the above notation, the quantity

$$
S=4 \frac{\left\|\Omega_{1} \Omega_{2}\right\|^{2}}{\left\|A_{1} A_{2}\right\|^{2}} \cdot\left(\overrightarrow{N_{1}} \cdot \overrightarrow{A_{1} A_{2}}\right) \cdot\left(\overrightarrow{N_{2}} \cdot \overrightarrow{A_{2} A_{1}}\right)
$$

is a polynomial in the input parameters, namely

$$
S=l^{2}\left(y_{2}{ }^{2} z_{2}{ }^{2}+z_{2}{ }^{4}\right)+m^{2}\left(y_{2}^{2}-z_{2}^{2}\right)^{2}+4 m n y_{2} z_{2}\left(y_{2}^{2}-z_{2}^{2}\right)+4 n^{2} y_{2}{ }^{2} z_{2}{ }^{2} .
$$

In addition, the following properties hold:

i) $4 \cdot S \cdot R^{2}=\left\|A_{1} A_{2}\right\|^{2}\left(S+l^{2} y_{2}^{2}\left(y_{2}^{2}+z_{2}^{2}\right)\right)$.

iii) the coordinates of $S \cdot \overrightarrow{\Omega M}$ are polynomials in the input parameters,

ii) the quantity $\left(\overrightarrow{\Omega_{1} \Omega_{2}} \cdot \overrightarrow{\Omega M}\right) \cdot\left(\overrightarrow{N_{1}} \cdot \overrightarrow{A_{1} A_{2}}\right) \cdot\left(\overrightarrow{N_{2}} \cdot \overrightarrow{A_{2} A_{1}}\right)$ is a polynomial in the input parameters, namely

$$
X\left(l y_{2}{ }^{2} z_{2}+l z_{2}{ }^{3}\right)+Y\left(-m y_{2}{ }^{2} z_{2}+m z_{2}{ }^{3}-2 n y_{2} z_{2}{ }^{2}\right)+Z\left(m y_{2}{ }^{3}-m y_{2} z_{2}{ }^{2}+2 n y_{2}{ }^{2} z_{2}\right) .
$$

This latter quantity provides a reduced equation of the supporting plane of the circle $\mathcal{C}$.

Case $\left(a_{2}\right)$. Now, consider the case where $\Omega_{1}$ is at infinity but not $\Omega_{2}$. Then the unique circle solution of the fitting problem is the intersection of the plane normal to $N_{1}$ and the sphere at $\Omega_{2}$, both passing through $A_{1}$. With the previously chosen notation for the frame and the coordinates, we now have $z_{2}=0, y_{2} \neq 0$ and $m \neq 0$. A straightforward computation shows that the equation of the plane containing the circle is $Z=0$ and in this plane the equation of the circle (multiplied by $4 m^{2}$ which is nonzero) is

$$
\left(2 m X-l y_{2}\right)^{2}+\left(2 m Y-y_{2} m\right)^{2}=m^{2} y_{2}^{2}+l^{2} y_{2}^{2} .
$$

Thus, its center $\Omega$ and the square of its radius $R$ are given by

$$
\Omega=\left(\frac{-l y_{2}}{2 m}, \frac{y_{2}}{2}, 0\right), \quad R^{2}=\frac{y_{2}^{2}\left(l^{2}+m^{2}\right)}{4 m^{2}} .
$$

We also notice that the quantity $S$ defined in Lemma 2, case $\left(a_{1}\right)$, specializes when $z_{2}=0$ to $S=m^{2} y_{2}^{4}$ which is nonzero. Actually, the specialization of the entire Lemma 2 is still valid so that it can always be applied when the 3D circle fitting problem admits a unique solution.

Therefore, case $\left(a_{2}\right)$ does not require a specific treatment compared to the case $\left(a_{1}\right)$ when dealing with explicit formulae.

Cases $(b)$ and $(c)$. In case $(b)$ there is no solution and this case can be detected with the following test: $z_{2}=0, y_{2} \neq 0$, and $m=0, l \neq 0$. In case $(c)$ there are infinitely many solutions and it can be detected with the following test : $m^{2}=2 \ln$ and $m \neq 0$; or $z_{2}=0$ and $l=m=0$.

We notice that the stronger condition " $m \neq 0$ and $m^{2} \neq 2 l n$ " excludes both cases $(b)$ and $(c)$ and only depends on the input normals $\left(N_{1}\right.$ and $\left.N_{2}\right)$. 


\section{Tori passing through a minimal point set}

A torus is defined as the set of points in the three-dimensional affine space $\mathbb{R}^{3}$ that are located at a fixed distance, called the small radius of the torus, of a given circle which is called the skeletal circle of the torus; the radius of this circle will be called the big radius of the torus and will be denoted by $R$, while the small radius will be denoted by $r$. Seven parameters are needed for a torus: six for its skeletal circle and an additional one for the small radius.

Below, we will prove our main theorem; i.e. we will solve the problem of computing the tori passing through three distinct points, two of them being oriented. These points form a minimal point set because they correspond to seven conditions. Thus, our input consists in three distinct points $P_{1}, P_{2}, P_{3}$ and two nonzero normal vectors $N_{1}, N_{2}$ that are attached to the points $P_{1}, P_{2}$.

Implicit equations of tori. The points of a torus are exactly those points that are at distance $r$, the small radius of the torus, to the skeletal circle of the torus, which is a $3 \mathrm{D}$ circle of center $\Omega$ and radius $R$.

We denote by $\vec{N}$ a normal vector to the supporting plane of the skeletal circle; a unitary normal vector is given by $\vec{N} /\|\vec{N}\|$. The squared distance of a point $M$ to the skeletal circle can be classically derived as

$$
\left(\frac{\vec{N}}{\|\vec{N}\|} \cdot \overrightarrow{\Omega M}\right)^{2}+\left(\sqrt{\|\Omega M\|^{2}-\left(\frac{\vec{N}}{\|\vec{N}\|} \cdot \overrightarrow{\Omega M}\right)^{2}}-R\right)^{2} .
$$

Expressing that this latter quantity must be equal to $r^{2}$ and squaring we obtain the following implicit equation of the torus:

$$
\left(\|\Omega M\|^{2}+R^{2}-r^{2}\right)^{2}=4 R^{2}\left(\|\Omega M\|^{2}-\left(\frac{\vec{N}}{\|\vec{N}\|} \cdot \overrightarrow{\Omega M}\right)^{2}\right)
$$

hence:

$$
\|\vec{N}\|^{2}\left(\|\Omega M\|^{2}+R^{2}-r^{2}\right)^{2}-4 R^{2}\left(\|\vec{N}\|^{2} \cdot\|\Omega M\|^{2}-(\vec{N} \cdot \overrightarrow{\Omega M})^{2}\right)=0
$$

Proof of the theorem. First, by a change of coordinates we can assume that $P_{1}$ is at the origin with $N_{1}=(0,0,1)$, and also that $P_{2}=\left(0, y_{2}, z_{2}\right)$. We set $N_{2}=(l, m, n)$ and $P_{3}=\left(x_{3}, y_{3}, z_{3}\right)$.

We introduce two new quantities $r_{1}$ and $r_{2}$ and we consider the two points $A_{1}=P_{1}+r_{1} N_{1}$ and $A_{2}=$ $P_{2}+r_{2} N_{2}$. These two points will belong to the skeletal circle of a torus, of small radius equal to $\left|r_{1}\right|$, that interpolates the two oriented points $P_{1}$ and $P_{2}$ providing

$$
r_{1}^{2}=r_{2}^{2}\left\|N_{2}\right\|^{2}=r_{2}^{2}\left(l^{2}+m^{2}+n^{2}\right) .
$$

Assume that we are in the first case of Proposition 1, i.e. the subcases $\left(a_{1}\right)$ or $\left(a_{2}\right)$, and so that there is a unique circle that interpolates the points $A_{1}$ and $A_{2}$ and which is normal to $N_{1}$ at $A_{1}$ and normal to $N_{2}$ at $A_{2}$. This circle is the skeletal circle of the interpolating torus. Taking again the notation of Section 2 , we denote by $\Omega=(a, b, c)$ its center, by $R$ its radius and by $\vec{N}:=(u, v, w)$ which is a normal vector to the supporting plane of this circle. From Equation (3), (1) and (2), the implicit equation of this interpolating torus can be written in terms of the space coordinates $x, y, z$ and of the parameters $y_{2}, z_{2}, l, m, n$ and $r_{1}, r_{2}$. It turns out that, thanks to the algebraic remarks summarized in Lemma 2 of the previous section, this equation can be factorized and replaced by a simplified polynomial that we will denote by $E$. To be more precise, assume that we are in the case $\left(a_{1}\right)$ and define the following polynomial quantities:

$$
\begin{gathered}
P:=\left\|A_{1} A_{2}\right\|^{2}=r_{2}^{2} l^{2}+m^{2} r_{2}^{2}+n^{2} r_{2}^{2}+2 m r_{2} y_{2}-2 n r_{1} r_{2}+2 n r_{2} z_{2}+r_{1}^{2}-2 r_{1} z_{2}+y_{2}^{2}+z_{2}^{2}, \\
Q_{1}:=\overrightarrow{N_{1}} \cdot \overrightarrow{A_{1} A_{2}}=n r_{2}-r_{1}+z_{2}, \quad Q_{2}:=\overrightarrow{N_{2}} \cdot \overrightarrow{A_{2} A_{1}}=l^{2} r_{2}+m^{2} r_{2}+n^{2} r_{2}+m y_{2}-n r_{1}+n z_{2} .
\end{gathered}
$$


Then, using the properties given in Lemma 2, straightforward symbolic computations show that the implicit equation of the torus (3) writes as

$$
\frac{P}{Q_{1}^{2} \cdot Q_{2}^{2}} \cdot E\left(x, y, z, x_{2}, y_{2}, l, m, n, r_{1}, r_{2}\right)
$$

where $E$ is a polynomial. Similar computations shows that this polynomial also encapsulates the case $\left(a_{2}\right)$. The polynomial equation $E=0$ is obviously of degree 4 in the variables $x, y, z$. It turns out that it is of degree 8 with respect to each of the variables $l, m$, of degree 6 with respect to each of the variables $n, y_{2}, z_{2}, r_{2}$ and of degree 7 with respect to the variable $r_{1}$.

Summarizing the above calculations, the tori that interpolate the three distinct points $P_{1}, P_{2}, P_{3}$, where $P_{1}$ are $P_{2}$ are oriented by $N_{1}$ and $N_{2}$ respectively, are given by the two algebraic equations

$$
E\left(x_{3}, y_{3}, z_{3}, x_{2}, y_{2}, l, m, n, r_{1}, r_{2}\right)=0
$$

(the variables $x, y, z$ have been substituted by the point $P_{3}$ in order to impose the interpolation condition at this point) and $U:=r_{1}{ }^{2}-r_{2}{ }^{2}\left(l^{2}+m^{2}+n^{2}\right)=0$ deduced from the condition (4). Since the polynomial $U$ is monic in $r_{1}$, we can perform an Euclidian division of $E$ by $U$ with respect to to variables $r_{1}$, which remainder is a polynomial in $r_{1}$ of degree at most 1 , that we denote by $S_{1}+r_{1} S_{2}$. Then, the system of equations finally reduces to

$$
S_{1}+r_{1} S_{2}=0, \quad r_{1}^{2}-r_{2}^{2}\left(l^{2}+m^{2}+n^{2}\right)=0
$$

where $S_{1}$ and $S_{2}$ are polynomials in $x_{3}, y_{3}, z_{3}, l, m, n, y_{2}, z_{2}, r_{2}$ of degree 4 and 3 respectively.

The elimination of $r_{1}$ from the two equations in the system (6) yields a polynomial in $x_{3}, y_{3}, z_{3}, l, m, n$, $y_{2}, z_{2}, r_{2}$ which is of degree 8 with respect to the variable $r_{2}$. Since the quantities $x_{3}, y_{3}, z_{3}, l, m, n, y_{2}, z_{2}$ are input data, one can solve $r_{2}$ from this degree 8 univariate polynomial and then build a unique torus for each of these values. Thus, we have proved the claimed theorem.

For the sake of completeness, we mention that the above elimination of $r_{1}$ in (6) can also be written as two univariate degree 4 polynomials. Indeed, the second equation in (6) shows that $r_{1}= \pm r_{2} \sqrt{l^{2}+m^{2}+n^{2}}$. Thus, this gives two cases and for each case $r_{1}$ can be substituted in the first equation of (6) to provide a degree 4 univariate polynomial in $r_{2}$.

To conclude this section, we mention that the upper bound of eight tori is reached on examples, even under the restriction of smooth tori. For instance, the following input data are interpolated by eight smooth tori, as show in Figure 2: $P_{1}=(0,0,0), N_{1}=(0,0,1), P_{2}=(0,-3.171791777,-2.369900007), N_{2}=$ $(-3.736353882,-2.170588024,0.215631583)$ and $P_{3}=(-0.394882587,3.246764454,-3.362188875)$. See also Figure 3 for another illustrative example.

\section{Effective solving and experiments}

In order to apply the previous method for the extraction of tori in 3D point clouds by means of RANSACbased approaches, we need to devise an algorithm that is fast and adapted to approximate data and numerical computations with double precision since this is the standard of the current libraries dealing with surface reconstruction.

The algebraic strategy we developed aims at producing closed form formulae that can be stored and used efficiently to solve the interpolation problem. Thus, the degree 8 polynomial in the variable $r_{2}$ we found (or the two degree 4 polynomials) could be pre-computed and its coefficients could be stored. Then, for each particular instance this polynomial could be solved and the tori extracted. However, the coefficients of this polynomial are big polynomials and their evaluation in double precision with approximate data yield significant numerical instability. In order to overcome this difficulty, we use a matrix-based formulation of the elimination of the variable $r_{1}$ in the system (6). More precisely, we form the Sylvester matrix of these two 

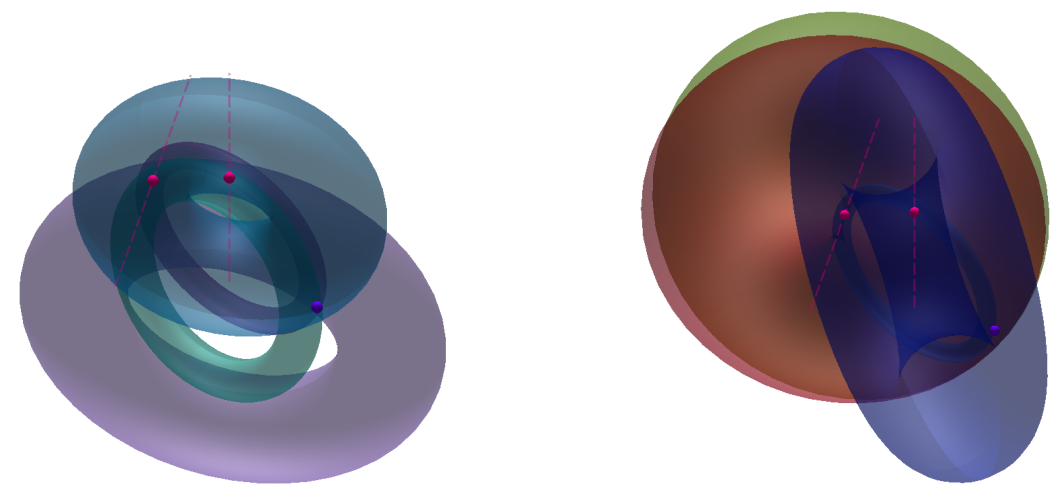

Figure 2: Eight tori that interpolate a minimal point set, separated into two groups of four torus in order to improve the visualization.

polynomials with respect to the variable $r_{1}$. This is a $3 \times 3$-matrix whose entries are polynomials of degree at most 4 in the variable $r_{2}$. Then, we compute its associated pencil of companion matrices $A, B$ which are $12 \times 12$ matrices. For each particular instance, the generalized eigenvalues and eigenvectors of these matrices are computed to provide the couple of roots $r_{1}, r_{2}$ of this system (see [1] or [2] for more details). In practice, the matrices $A$ and $B$ are pre-computed and stored. It turns out that their entries are of smaller size and degree compared to the coefficients of the above degree 8 polynomial in $r_{2}$. This and the use of generalized eigenvalues yield a much more numerically stable algorithm.

A prototype of the interpolation algorithm we described above has been implemented in ${\mathrm{C}++^{*}}^{*}$, with the help of the computer algebra software Maple for computing the matrix-based closed formulae. We observed that the extraction of the tori from a random set of three points, two of them being oriented, is very fast and that its cost is almost constant independently of the point set, about $0.2 \mathrm{~ms}$. Contrary to the usual approaches (e.g. [3, 4, 7]), our method may output several tori for a given point set, up to eight tori. Nevertheless, we observed that less than four tori are obtained in $97 \%$ of cases in average. In practice, the reduction of the number of tori, typically to select only one or no torus, can also be done by taking into account the additional information of the normal at the third point $P_{3}$ if available. Indeed, it might be the case that this normal is expected to be in a certain cone of tolerance and hence the tori we computed can be filtered by comparing the orientation of $P_{3}$ for each torus with this cone.

\section{Conclusion}

This work continues our study of optimized basic shapes interpolation from the smallest possible set of mixed oriented points. We presented a point of view which combines geometric and algebraic aspects. The minimal (mixed) point set for defining a finite number of tori consists in two oriented points and a simple one, corresponding to seven numbers of freedom. We proposed an original analysis and an efficient algorithm for performing this extraction. Its main step consists of computing the generalized eigenvalues of a pre-computed pencil of matrices in closed-form.

\section{References}

[1] Laurent Busé, André Galligo, and Jiajun Zhang. Extraction of cylinders and cones from minimal point sets. Graphical Models, 86:1-12, 2016.

*ASurfExt C++ library :https://gitlab.inria.fr/lbuse/ASurfExt/wikis/home 

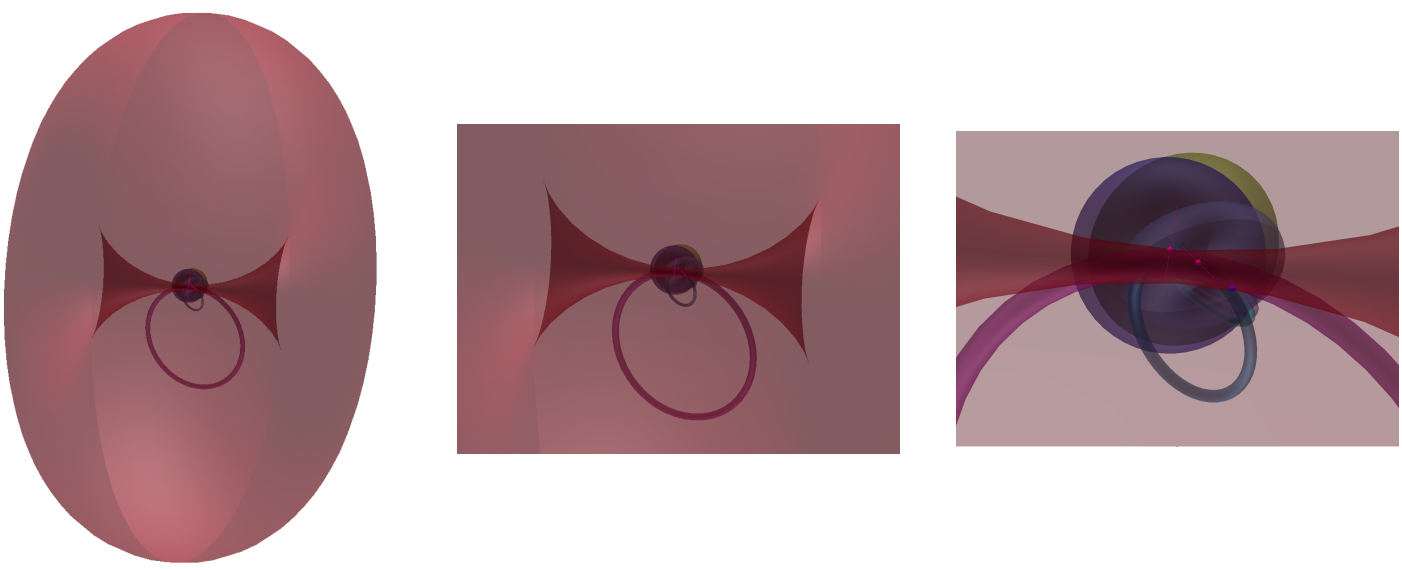

Figure 3: Eight smooth tori that interpolate a point set. The focus is increased from left to right in order to visualize all the eight tori.

[2] Laurent Busé, Houssam Khalil, and Bernard Mourrain. Resultant-based methods for plane curves intersection problems. In Victor G. Ganzha, Ernst W. Mayr, and Evgenii V. Vorozhtsov, editors, Computer Algebra in Scientific Computing (CASC), volume 3718, pages 75-92, Kalamata, Greece, September 2005. Springer Berlin / Heidelberg.

[3] Martin A. Fischler and Robert C. Bolles. Random sample consensus: A paradigm for model fitting with applications to image analysis and automated cartography. Commun. ACM, 24(6):381-395, June 1981.

[4] John R. Kender and Rick Kjeldsen. On seeing spaghetti: A novel self-adjusting seven parameter hough space for analyzing flexible extruded objects. In Proceedings of the 12th International Joint Conference on Artificial Intelligence - Volume 2, IJCAI'91, pages 1271-1277, San Francisco, CA, USA, 1991. Morgan Kaufmann Publishers Inc.

[5] John R. Kender and Rick Kjeldsen. On seeing spaghetti: Self-adjusting piecewise toroidal recognition of flexible extruded objects. IEEE Trans. Pattern Anal. Mach. Intell., 17(2):136-157, 1995.

[6] Gabor Lukács, Ralph Martin, and Dave Marshall. Faithful least-squares fitting of spheres, cylinders, cones and tori for reliable segmentation. In Hans Burkhardt and Bernd Neumann, editors, Computer Vision - ECCV'98, volume 1406 of Lecture Notes in Computer Science, pages 671-686. Springer Berlin Heidelberg, 1998.

[7] Ruwen Schnabel, Roland Wahl, and Reinhard Klein. Efficient ransac for point-cloud shape detection. Computer Graphics Forum, 26(2):214-226, June 2007.

[8] Zahra Toony, Denis Laurendeau, and Christian Gagné. Pgp2x: Principal geometric primitives parameters extraction. In Proc. of the 10th International Conference on Computer Graphics Theory and Applications (GRAPP), 2015. 